\title{
Infrared induced visible emission from porous silicon: the mechanism of anodic oxidation
}

\author{
E.S. Kooij *, A.R. Rama, J.J. Kelly \\ Department of Condensed Matter, Debye Institute, Utrecht University, P.O. Box 80000, 3508 TA Utrecht, The Netherlands \\ Received 1 May 1996; accepted for publication 22 July 1996
}

\begin{abstract}
The visible luminescence caused by anodic oxidation of $p$-type porous silicon has been studied. It is shown that similar luminescence can be observed in $n$-type material by illumination with near-infrared light. Addition of a suitable reducing agent to the electrolyte solution can both suppress the oxidation of the porous layer and quench its luminescence. These results confirm a previously suggested mechanism, in which the capture of a valence band hole in a surface bond of the porous semiconductor gives rise to a surface state intermediate capable of thermally injecting an electron into the conduction band.
\end{abstract}

Keywords: Electrochemical methods; Electroluminescence; Models of surface chemical reactions; Oxidation; Porous silicon; Semiconductor-electrolyte interfaces

\section{Introduction}

Various types of electroluminescence (EL) have been reported for porous silicon in contact with an aqueous solution. To generate EL, minority carriers have to be injected into the semiconductor. Radiative recombination of the injected charge carriers with the majority carriers leads to light emission, characteristic of the semiconductor. Efficient visible EL has been observed from $n$-type porous silicon in contact with an electrolyte containing strong oxidizing agents like $\mathrm{S}_{2} \mathrm{O}_{8}^{2-}$ and $\mathrm{H}_{2} \mathrm{O}_{2}[1,2]$. It is assumed that holes are injected into the valence band by radical ions which are formed in the first step of the reduction of these species. Recently, we showed that EL could be generated in a similar way by injecting electrons

* Corresponding author. into the conduction band of $p$-type porous silicon [3]. The methylviologen radical cation, which is formed in the first step of the reduction of divalent methylviologen, is a strong reducing agent with energy levels overlapping the conduction band of silicon [4].

Bright visible luminescence has also been observed during the anodic oxidation of porous silicon in HF-free indifferent electrolyte solutions [5-13]. Most of the work has concentrated on the electrochemical oxidation of $p$-type material $[5-8,10,12,13]$. The process responsible for the minority carrier injection is not clear as there are no electroactive species in solution capable of donating electrons to the conduction band. It has been suggested that $\mathrm{Si}-\mathrm{H}$ species, present at high density on the hydrogen-terminated surface of the porous structure, are the source of the electrons $[5,7,9]$. The oxidation of $\mathrm{Si}-\mathrm{H}$ to $\mathrm{Si}-\mathrm{OH}$ could 
lead to electron injection into the conduction band, which can be represented by

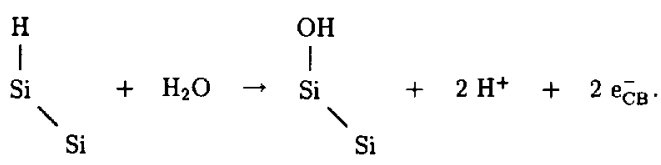

For the sake of simplicity only one $\mathrm{Si}-\mathrm{Si}$ back bond and one surface species are shown in Eq. (1). The transient nature of the current and of the corresponding luminescence is attributed to the fact that $\mathrm{Si}-\mathrm{H}$ species are consumed in the oxidation process and are not regenerated. Recently, Uosaki et al. [13] reported in situ FT-IR measurements which clearly show that the anodic oxidation and the corresponding luminescence are accompanied by a strong decrease of the $\mathrm{Si}-\mathrm{H}$ coverage. However, Hory et al. [14] found the $\mathrm{Si}-\mathrm{H}$ coverage to remain approximately constant, which might be due to a much smaller anodization current used in the experiments. On the basis of UPS measurements by Landemark et al. [15] direct electron injection would, however, seem unlikely as the energy levels corresponding to $\mathrm{Si}-\mathrm{H}$ surface species are located well below the valence band edge. This is supported by theoretical calculations [16]. Consequently, one would expect the surface species to undergo interaction with the valence band, i.e. hole capture, instead of with the conduction band. As we will show, no anodic dark current is found with $n$-type porous silicon electrodes, which also argues against reaction (1) being responsible for the anodic oxidation.

Recently, our group has shown that electroluminescence can be observed during anodic dissolution of p-type InP and GaAs electrodes [17]. It was concluded that holes localized in surface bonds give rise to dissolution intermediates with energy levels in the bandgap of the semiconductor. Electrons can be thermally excited from such states into the conduction band and recombine radiatively with valence band holes, giving rise to band-band emission. A similar type of reaction has also been suggested as a possible source of anodic luminescence in $p$-type porous silicon $[7,8,11,12]$. Electroluminescence of InP was quenched when a reducing agent $\left(\mathrm{Fe}^{2+}\right)$, capable of suppressing the anodic dissolution reaction, was present in solution.

The aim of the present study was to elucidate the mechanism of the anodic oxidation of porous silicon and of the corresponding light emission. We wanted to show that valence band holes are essential for initiating both processes. In the case of $p$-type electrodes the holes needed for the generation of EL are supplied from the substrate. Holes can be generated in $n$-type porous silicon by illuminating with visible light corresponding to the bandgap of the porous semiconductor. This, however, gives rise to photoluminescence which is difficult to distinguish from the emission resulting from anodic oxidation. We tried instead to inject holes from the substrate. For this purpose we used nearinfrared light with an energy greater than the bandgap of bulk silicon; this light was not absorbed by the wider bandgap porous silicon layer. In this way it proved possible to oxidize the $n$-type porous silicon. The importance of valence band holes for anodic oxidation and luminescence is further supported by the results of experiments in which an $\mathrm{Fe}(\mathrm{CN})_{6}^{4-}$ salt in solution was used to suppress the anodic reaction. Madou et al. [18] have shown that $\mathrm{Fe}(\mathrm{CN})_{6}^{4-}$ ions can effectively compete with the anodic oxidation of crystalline silicon, thereby "stabilizing" the electrode. On the basis of these measurements we discuss the mechanism of the anodic oxidation and luminescence of porous silicon.

\section{Experimental}

Electrodes were cut with a diamond saw from $n$-type $(1$ to $10 \Omega \cdot \mathrm{cm}$ ) and $p$-type $(1$ to $15 \Omega \cdot \mathrm{cm}$ ) single crystal silicon wafers which had the $(100)$ orientation. Crystalline silicon electrodes were dipped in a $1 \mathrm{M} \mathrm{HF}, 2 \mathrm{M} \mathrm{NH} \mathrm{NH}_{4} \mathrm{~F}$ solution for 1 minute before each measurement to remove surface oxides. Porous silicon electrodes were formed by anodic etching for 4 minutes in a 1:1 mixture of 40\% HF: ethanol. The current densities were $35 \mathrm{~mA} \mathrm{~cm} \mathrm{~cm}^{-2}$ and $18 \mathrm{~mA} \mathrm{~cm}{ }^{-2}$ for $p$-type and $n$ type silicon, respectively, giving $6 \mu \mathrm{m}$ and $3 \mu \mathrm{m}$ thick porous layers for the two types of material, both with a porosity of approximately $80 \%$. A two- 
electrode configuration with a Pt counter electrode was used. Anodization was performed under illumination at $0.5 \mathrm{~V}$ cell voltage for $n$-type and galvanostatically in the dark for $p$-type samples. Before and after each measurement the presence of the luminescent porous layer was checked by a PL measurement at an excitation wavelength of $354 \mathrm{~nm}$.

The electrochemical experiments were performed using an EG and G Princeton Applied Research (PAR) 366A bipotentiostat in a conventional electrochemical cell containing a Pt counter electrode and a saturated calomel electrode (SCE) as reference. All potentials are given with respect to SCE. For most of the measurements using silicon disc electrodes the electrode surface was defined with an O-ring in a PCTFE (Kel-F) holder; the exposed area was $0.38 \mathrm{~cm}^{2}$. In some cases, which will be indicated in the text, the semiconductor disc was mounted in epoxy resin and the electrode surface was in this case not screened from the solution at the edges of the wafer; the electrode area of these samples was $0.12 \mathrm{~cm}^{2}$. For experiments with the rotating ring-disc electrode ( $R R D E$ ) configuration we used a silicon disc ( $4.0 \mathrm{~mm}$ diameter) as the working electrode. A Au ring $(4.3 \mathrm{~mm}$ and $6.4 \mathrm{~mm}$ inner and outer diameter, respectively) was used to detect products formed at the disc. A collection efficiency of 0.50 was calculated from these dimensions [19].

The luminescence was measured using a standard silicon photodiode in combination with an Oriel Current Preamplifier (Model 70710) or with a Perkin-Elmer MPF-44B Fluorescence Spectrometer or an Oriel Instaspec IV CCD detection system. For illumination either a white light source (Schott KL1500) or an infrared LED (Siemens, $\lambda_{\max }=950 \mathrm{~nm}, \Delta \lambda_{\text {FWHM }}=55 \mathrm{~nm}$ ) was used. The measurements were carried out at room temperature. The $\mathrm{pH}$ of all solutions was between 6 and 7. The chemicals were of p.a. grade (Merck). Before each measurement nitrogen gas was bubbled through the solution to remove oxygen.

\section{Results}

In this section results are presented for the oxidation of crystalline and porous silicon electrodes. Measurements on $p$-type material in the potentiostatic and the galvanostatic regime are first described. The effect of ferrocyanide on the oxidation and on the emission characteristics will be shown. After this, results are presented for the photoanodic oxidation of $n$-type electrodes and the emission from $n$-type porous silicon under illumination with infrared light. Again, the effect of the stabilizing agent will be considered. Finally, we describe the influence of the method of mounting the electrodes on the current-potential and emission-potential characteristics.

\section{1. p-type electrodes}

In Fig. 1 the current-potential curve of a $p$-type crystalline silicon electrode in $1 \mathrm{M} \mathrm{KCl}$ solution is shown. The potential was scanned from $0 \mathrm{~V}$ to 3 $\mathrm{V}$ and back at a rate of $50 \mathrm{mV} / \mathrm{s}$. Current onset is generally found at around $0.2 \mathrm{~V}$, followed by a maximum at approximately $0.75 \mathrm{~V}$. In some cases an extra current shoulder was obtained about 0.3 $\mathrm{V}$ more negative than the main peak, but its height was not very reproducible. After the maximum the current decreases to an almost potential-independent value. The height of both this plateau and the current maximum depend on the scan rate, while the position of the maximum shifts to more positive potentials as the scan rate increases. Since the current decreases in the return scan towards negative potentials and the peak is absent in subsequent scans, we conclude that a passivating oxide layer is formed on the electrode surface. The total

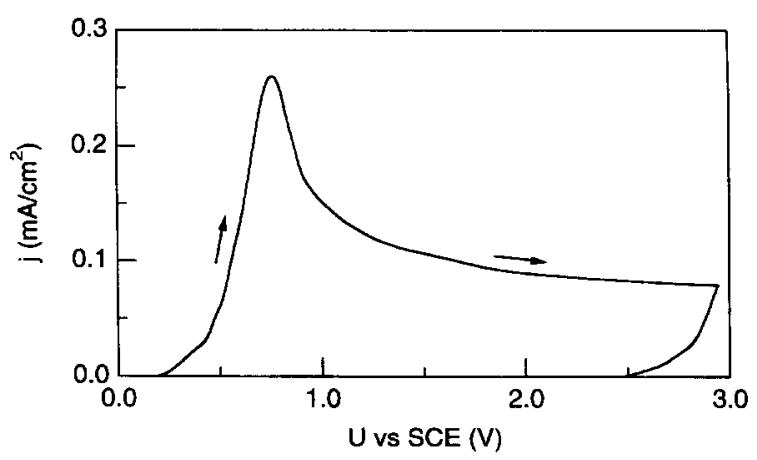

Fig. 1. Current-potential curve of a stationary $p$-type crystalline silicon electrode in $1 \mathrm{M} \mathrm{KCl}$ solution under potentiostatic control. The potential was scanned at a rate of $50 \mathrm{mV} / \mathrm{s}$. 
charge under the anodic peak is approximately 1.8 $\mathrm{mC} \mathrm{cm}{ }^{-2}$. If four charge carriers are required for the oxidation of each silicon atom and the density of the oxide is taken to be the same as that of thermal oxide, then this charge corresponds to a layer with a thickness of about $1.4 \mathrm{~nm}$. The oxide continues to grow as long as the potential is scanned in the positive direction.

Fig. $2 \mathrm{a}$ shows the current-potential curve of a $p$-type porous electrode in $1 \mathrm{M} \mathrm{KCl}$ solution. The potential was again scanned from $0 \mathrm{~V}$ to $3 \mathrm{~V}$ and back at a rate of $50 \mathrm{mV} / \mathrm{s}$. Characteristics similar to those obtained with non-porous electrodes are observed: a current maximum is found at $0.75 \mathrm{~V}$ after which the current decreases to an almost potential-independent value comparable to that of Fig. 1. However, the peak current of the porous electrode is about one order of magnitude higher, while the charge under the peak amounts to approximately $14 \mathrm{mC} \mathrm{cm}^{-2}$. The shoulder of the current peak at negative potentials also seems to
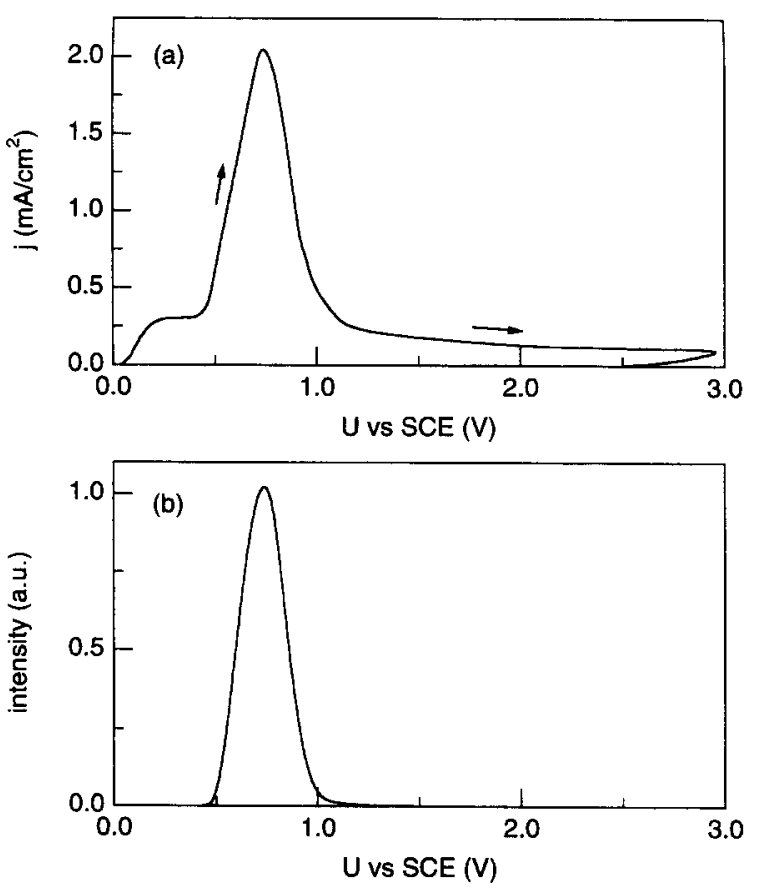

Fig. 2. Current-potential (a) and emission-potential (b) curves of a stationary p-type porous silicon electrode in $1 \mathrm{M} \mathrm{KCl}$ solution under potentiostatic control. The potential was scanned at a rate of $50 \mathrm{mV} / \mathrm{s}$. be larger with the porous electrode, although the current at $0.25 \mathrm{~V}$ is not always as pronounced as that shown in Fig. 2a. The luminescence measured during the oxidation of the porous silicon electrode is shown in Fig. 2b. Strong visible emission, which can be observed by the naked eye in slightly subdued light, is found in the same potential range in which the current maximum is measured. The luminescence is only detected during the main oxidation peak; the current shoulder at around $0.25 \mathrm{~V}$ does not cause emission. An orange to red glow is emitted uniformly by the whole electrode surface. After the peak the emission vanishes at potentials at which the current shows a plateau. In the return scan and in subsequent scans no emission could be detected.

In Fig. 3 a current-potential curve is shown for a $p$-type porous electrode in $0.1 \mathrm{M} \mathrm{K} \mathrm{K}_{4} \mathrm{Fe}(\mathrm{CN})_{6}$, $1 \mathrm{M} \mathrm{KCl}$ solution. When the potential is scanned from $0 \mathrm{~V}$ to $3 \mathrm{~V}$ a large current peak is observed. The maximum current is significantly higher than that observed in indifferent electrolyte solution and the peak is much broader. The charge under the peak $\left(\approx 250 \mathrm{mC} \mathrm{cm}^{-2}\right)$ is much larger than that measured in the absence of $\mathrm{Fe}(\mathrm{CN})_{6}^{4-}(14 \mathrm{mC}$ $\left.\mathrm{cm}^{-2}\right)$. Clearly, the oxidation of $\mathrm{Fe}(\mathrm{CN})_{6}^{4-}$ is competing with the oxidation of silicon. Nevertheless, the electrode is eventually passivated because of diffusion limitation in the supply of $\mathrm{Fe}(\mathrm{CN})_{6}^{4-}$ to the surface. The current decreases at more positive potentials and remains small in subsequent scans. Ferrocyanide efficiently quenches the luminescence

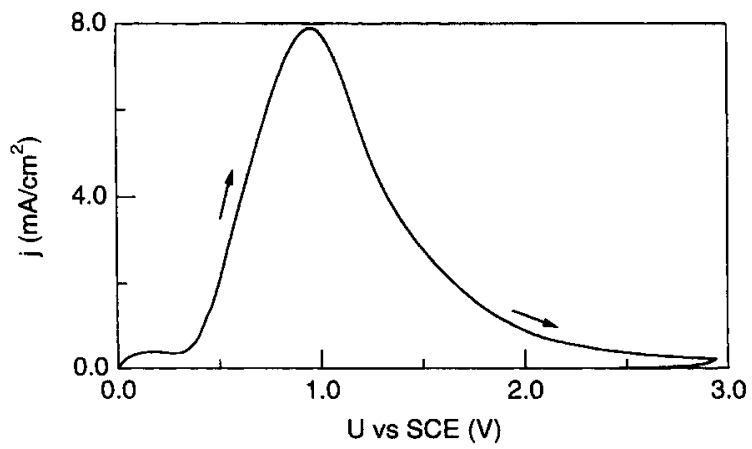

Fig. 3. Current-potential curve of a stationary p-type porous silicon electrode in $0.1 \mathrm{M} \mathrm{K}_{4} \mathrm{Fe}(\mathrm{CN})_{6}, 1 \mathrm{M} \mathrm{KCl}$ solution under potentiostatic control. The potential was scanned at a rate of $50 \mathrm{mV} / \mathrm{s}$. 
as no emission was detected during the current-potential measurements of Fig. 3. It should be pointed out that the redox system did not quench the photoluminescence of the porous layer.

With $p$-type porous electrodes in $1 \mathrm{M} \mathrm{KCl}$ solution under galvanostatic operation the potential-time plots of Fig. 4a were obtained at two different current densities. When the current is switched on, the potential rises quickly to values of $1.2 \mathrm{~V}$ and $2.0 \mathrm{~V}$ for current densities of $2.5 \mathrm{~mA}$ $\mathrm{cm}^{-2}$ and $12.5 \mathrm{~mA} \mathrm{~cm}^{-2}$, respectively. The potential then remains approximately constant for some time, after which it increases strongly. The point at which the potential increases depends on the applied current density, but the total charge which passes through the electrode is approximately the same for different current densities $(\approx 300 \mathrm{mC}$ $\mathrm{cm}^{-2}$ ). This value is much larger than that obtained from the potentiodynamic measurement of Fig. 2a. The oxidized fraction of the porous silicon layer
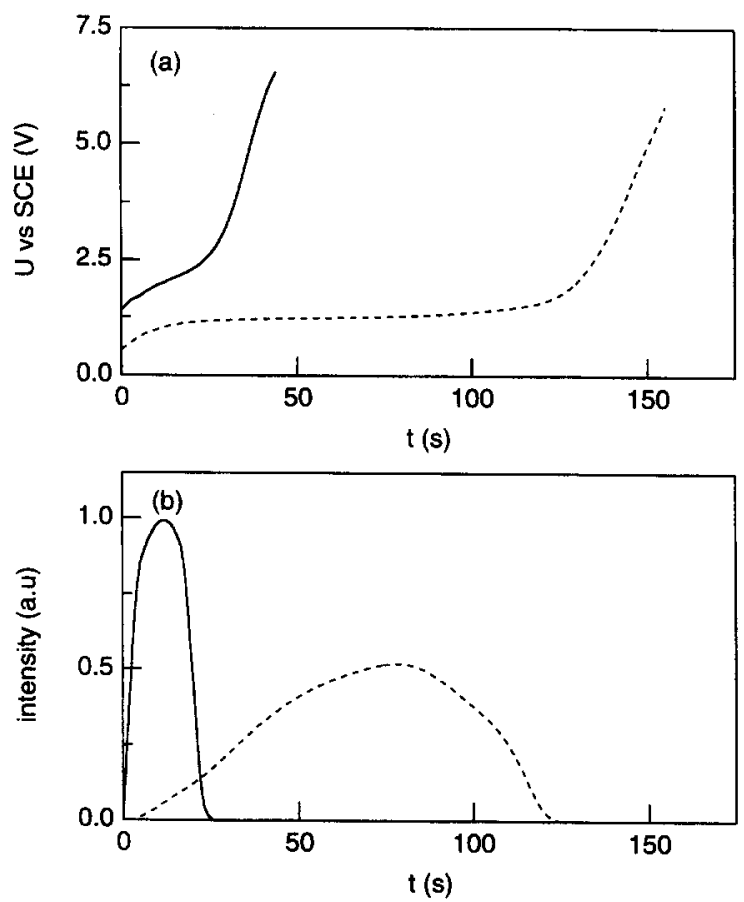

Fig. 4. Time-dependence of the potential (a) and the emission (b) of a stationary $p$-type porous silicon electrode in $1 \mathrm{M} \mathrm{KCl}$ solution under galvanostatic control. The curves obtained using current densities of $12.5 \mathrm{~mA} \mathrm{~cm} \mathrm{~cm}^{-2}$ and $2.5 \mathrm{~mA} \mathrm{~cm} \mathrm{~cm}^{-2}$ are given by the solid and the dashed line, respectively. amounts to about $10 \%$. While the potential is approximately constant, strong visible emission is found with a time dependence as shown in Fig. 4b. At lower current densities the emission lasts longer but its maximum intensity is lower. Similar results have been reported by other workers $[5-8,10,12]$. The point at which the emission intensity decreases to zero corresponds to the time at which the potential starts to increase. This effect has been attributed to formation of oxide at the porous silicon/substrate interface; the porous structure becomes isolated and the charge carriers can no longer reach the luminescent regions within the porous layer [6]. This would also account for the incomplete oxidation of the porous layer mentioned above for the potentiodynamic experiments. We have also measured the time-dependence of the emission spectrum. Results similar to those found by Billat [12] and Uosaki et al. [13] were obtained; the emission maximum shifts significantly towards shorter wavelengths as the oxidation time increases. This blue-shift was explained by assuming a size-distribution of the light-emitting particles; larger particles are oxidized first and the smaller particles become active as the oxidation proceeds.

When we repeated the experiment of Fig. 4a using the same $p$-type porous electrode in the presence of $\mathrm{Fe}(\mathrm{CN})_{6}^{4-}$, we obtained the potential-time curves shown in Fig. 5 at two current densities. In contrast to what we found in

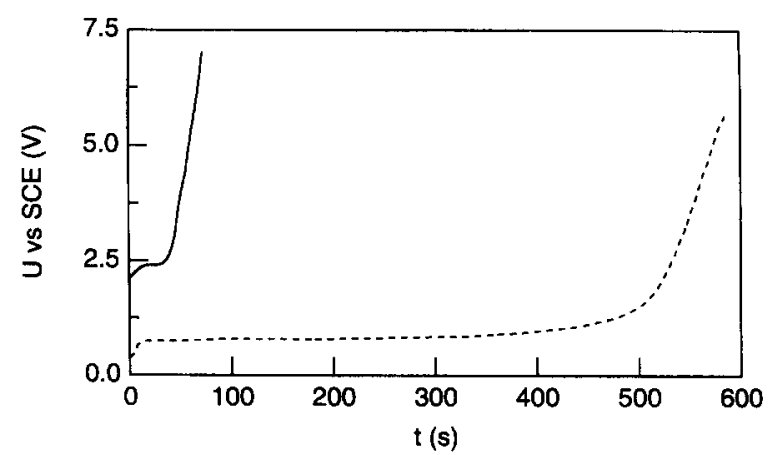

Fig. 5. Time-dependence of the potential of a stationary p-type porous silicon electrode in $0.1 \mathrm{M} \mathrm{K}_{4} \mathrm{Fe}(\mathrm{CN})_{6}, 1 \mathrm{M} \mathrm{KCl}$ solution under galvanostatic control. The curves obtained using current densities of $12.5 \mathrm{~mA} \mathrm{~cm} \mathrm{~cm}^{-2}$ and $2.5 \mathrm{~mA} \mathrm{~cm} \mathrm{~cm}^{-2}$ are given by the solid and the dashed line, respectively. 
indifferent electrolyte solutions, the potential remains constant for a longer period which depends on the current density and on the concentration of $\mathrm{Fe}(\mathrm{CN})_{6}^{4-}$. No light emission is observed in either case. This indicates that there is competition between $\mathrm{Fe}(\mathrm{CN})_{6}^{4-}$ oxidation and silicon oxidation. If the current density is low $(0.1-0.5 \mathrm{~mA}$ $\left.\mathrm{cm}^{-2}\right)$ and the $\mathrm{Fe}(\mathrm{CN})_{6}^{4-}$ concentration is high $(0.5 \mathrm{M})$, the potential remains constant for a very long period (up to 30 minutes) and the luminescence is quenched. On the other hand if the $\mathrm{Fe}(\mathrm{CN})_{6}^{4-}$ concentration is low and the current density is high then the silicon becomes oxidized and luminescence is detected. Mass transport of $\mathrm{Fe}(\mathrm{CN})_{6}^{4-}$ to the electrode surface is insufficient to prevent oxidation.

That $\mathrm{Fe}(\mathrm{CN})_{6}^{4-}$ is indeed oxidized to $\mathrm{Fe}(\mathrm{CN})_{6}^{3-}$ could be verified using the rotating $\mathrm{Au} / p$-type porous silicon ring-disc configuration. At the disc the current may be due to oxidation of both silicon and $\mathrm{Fe}(\mathrm{CN})_{6}^{4-}$. The $\mathrm{Fe}(\mathrm{CN})_{6}^{3-}$ formed at the disc is reduced at the ring and detected as a cathodic current. Fig. 6 shows a measurement in $0.1 \mathrm{M} \mathrm{K}_{4} \mathrm{Fe}(\mathrm{CN})_{6}, 1 \mathrm{M} \mathrm{KCl}$ solution. The ratio of ring current $i_{\text {ring }}$ to disc current $i_{\text {disc }}$ is plotted as a function of the disc potential, for a constant ring potential of $-0.5 \mathrm{~V}$. The rotation rate of the RRDE was $1000 \mathrm{rpm}$. The fact that the $i_{\text {ring }} / i_{\text {disc }}$ ratio is close to the calculated collection efficiency of 0.50 shows that the reaction occurring at the disk is almost exclusively the oxidation of

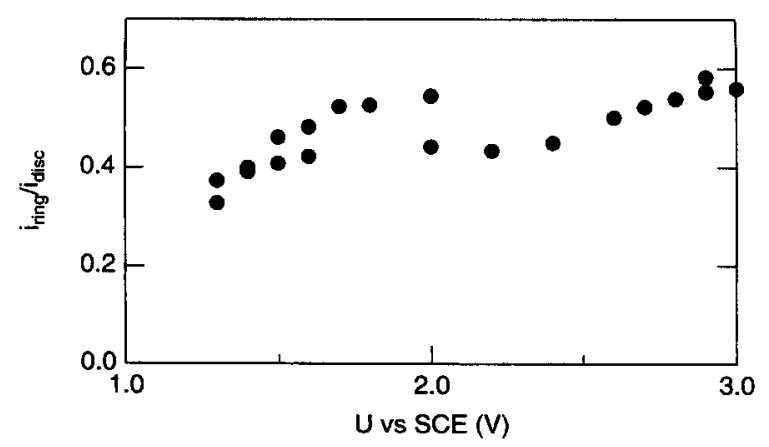

Fig. 6. Ratio of the ring current to the disc current of a $\mathrm{Au} / \mathrm{p}$ type porous silicon RRDE as a function of the disc potential in $0.1 \mathrm{M} \mathrm{K} \mathrm{K}_{4} \mathrm{Fe}(\mathrm{CN})_{6}, 1 \mathrm{M} \mathrm{KCl}$ solution. The ring potential was kept at a constant value of $-0.5 \mathrm{~V}$, while the electrode was rotated at $1000 \mathrm{rpm}$.
$\mathrm{Fe}(\mathrm{CN})_{6}^{4-}$ and that stabilization of silicon indeed occurs.

\section{2. n-type electrodes}

The result shown in Fig. 7 was obtained with $n$ type crystalline silicon in indifferent electrolyte solution $(1 \mathrm{M} \mathrm{KCl})$. The dashed line gives the current-potential curve in the dark. The current increases only slightly as the potential is increased; significant oxidation does not occur as this current-potential curve is observed in many subsequent scans. However, when we illuminate the electrode with white light an anodic photocurrent is observed. At lower light intensities a potentialindependent current is found, whose magnitude depends on the photon flux (not shown). At higher light intensity, features are observed similar to those of the p-type electrode in the dark (Fig. 7); a current maximum in the first scan towards positive potentials leads to passivation, after which the current decreases to a value comparable to that shown in Fig. 1 and Fig. 2. In the return scan the current rapidly decreases to a small value and a current peak is not found in subsequent scans.

In Fig. 8a the current density of an infrared illuminated $n$-type porous silicon electrode, polarized at $1.5 \mathrm{~V}$ versus SCE, is shown as a function of time. When the light is turned on, the current is constant until about $40 \mathrm{mC} \mathrm{cm}{ }^{-2}$ has passed through the electrode, after which passivation of

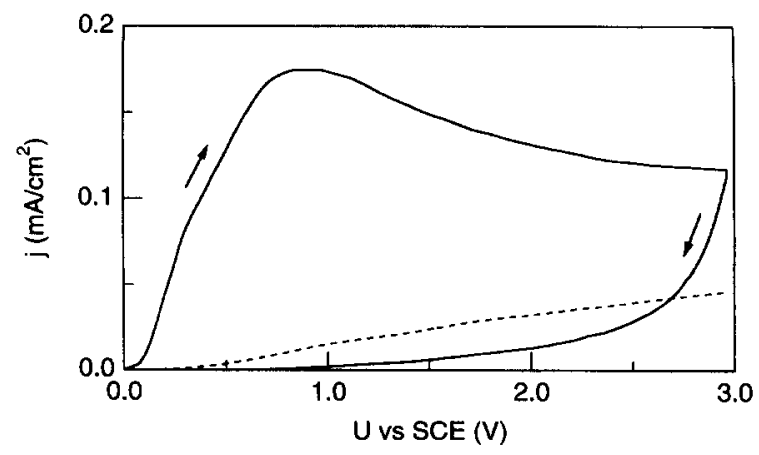

Fig. 7. Current--potential curve of a stationary $n$-type crystalline silicon electrode in $1 \mathrm{M} \mathrm{KCl}$ solution under potentiostatic control. The curves obtained in the dark and under illumination are given by the dashed and the solid line, respectively. The potential was scanned at a rate of $50 \mathrm{mV} / \mathrm{s}$. 

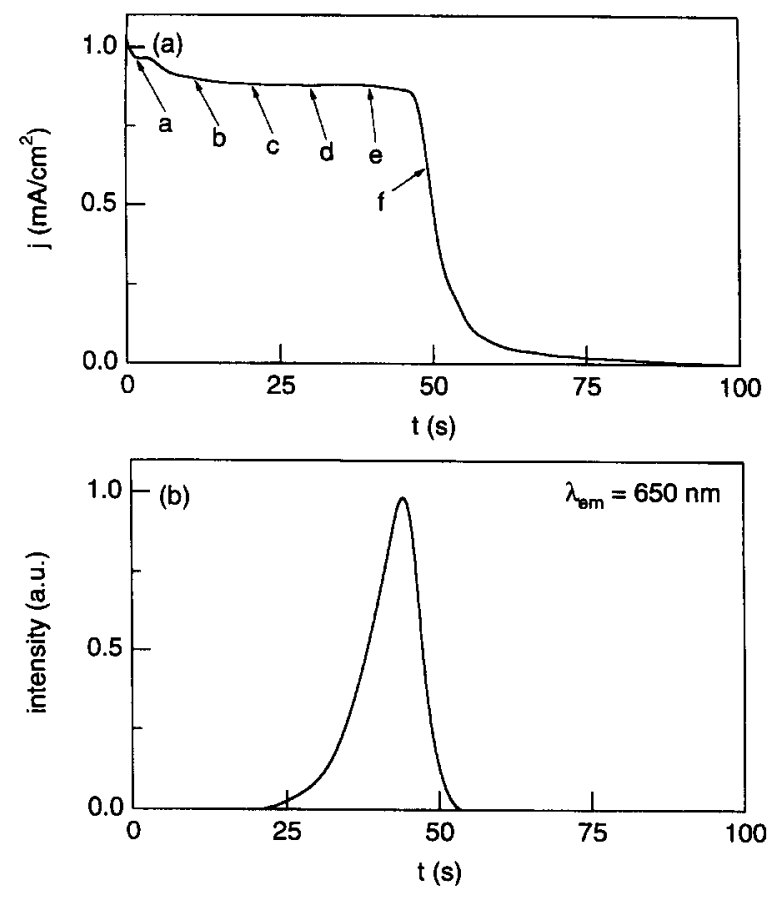

Fig. 8. Time-dependence of the current (a) and the emission (b) of a stationary $n$-type porous silicon electrode in $1 \mathrm{M} \mathrm{KCl}$ solution polarized at $1.5 \mathrm{~V}$, under illumination with infrared light. The letters a-f indicate when the spectra in Fig. 9 were recorded.

the electrode causes the current to decrease. The height of the plateau was linearly dependent on the photon flux, while the duration was inversely proportional to the light intensity, indicating that the oxide layer has approximately the same thickness at different light intensities. The fact that the charge involved in the electrochemical oxidation of $n$-type samples is lower than that required for $p$-type samples may be due to the difference in morphology of porous layers formed on $n$-type and p-type substrates [20].

During illumination with infrared light a uniform red glow from the electrode surface is clearly visible in the dark. The emission measured at $650 \mathrm{~nm}$ using a monochromator and a photomultiplier is shown in Fig. 8b as a function of time. The luminescence intensity shows a maximum during the second half of the current plateau. When the current decreases due to formation of the oxide layer, the emission also disappears. Apparently, the luminescent regions become electrically isolated

from the substrate and charge carriers are not able to reach the light-emitting regions in the porous structure. As in the case of $p$-type electrodes in the dark, $\mathrm{Fe}(\mathrm{CN})_{6}^{4-}$ also quenches the infrared generated luminescence of $n$-type porous silicon. The spectrum of the emission in Fig. $8 \mathrm{~b}$ depends on the oxidation time as is shown in Fig. 9. The large increase in intensity at longer wavelengths is due to reflection at the electrode surface of the light emitted by the infrared source. As the oxidation proceeds, the emission at wavelengths below $830 \mathrm{~nm}$ first increases, while the maximum clearly shifts towards shorter wavelengths. This blue-shift is similar to that observed with $p$-type electrodes $[12,13]$. At the end of the current plateau in Fig. $8 \mathrm{a}$ the emission strongly decreases, similar to what is shown in Fig. $8 \mathrm{~b}$.

As mentioned in the experimental section the electrodes were mounted in two different ways. When an epoxy resin was used, the electrode edges, which may be mechanically damaged on cutting the wafers, were exposed to the solution. This was not the case when an O-ring was used to define the electrode; only the surface of the wafer was exposed. The effect of edge damage on the electrochemical results for $n$-type porous electrodes is shown in Fig. 10. The potential of both an O-ring electrode (dashed line) and an electrode sealed with epoxy resin (solid line) was scanned in the dark in $1 \mathrm{M} \mathrm{KCl}$ solution from $0 \mathrm{~V}$ to $3 \mathrm{~V}$ at a rate of 50 $\mathrm{mV} / \mathrm{s}$. The current found with the porous O-ring

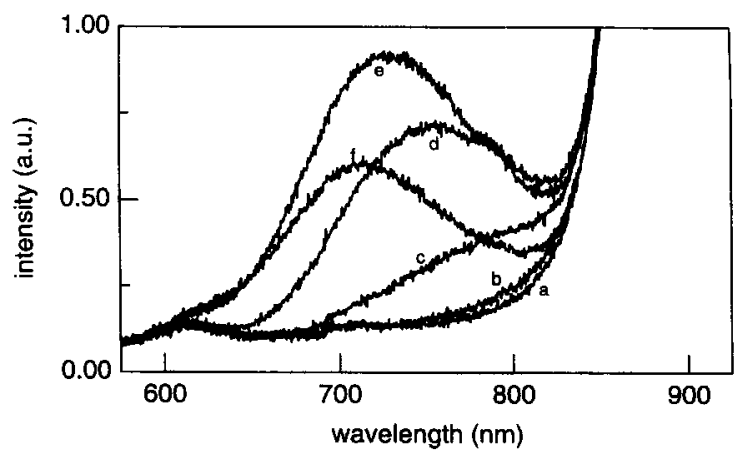

Fig. 9. Time-dependence of the emission spectrum during the anodic oxidation of a stationary $n$-type porous silicon electrode in $1 \mathrm{M} \mathrm{KCl}$ solution polarized at $1.5 \mathrm{~V}$, under illumination with infrared light. The time at which the spectra were recorded is indicated in Fig. 8 with the letters a-f. 

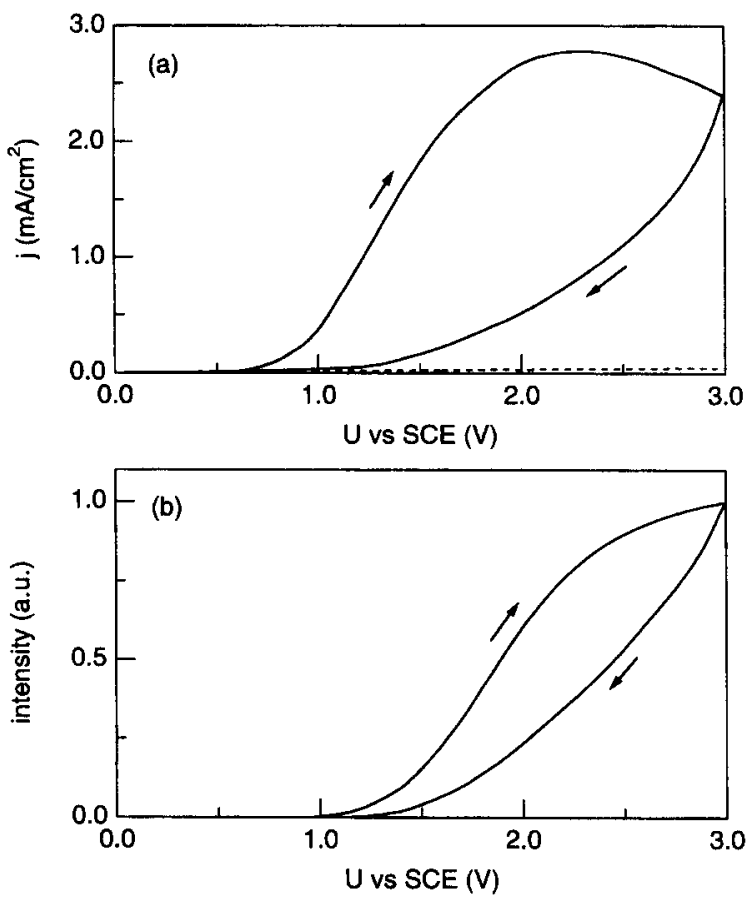

Fig. 10. Current-potential (a) and emission-potential (b) curves of two different stationary porous silicon electrodes in $1 \mathrm{M} \mathrm{KCl}$ under potentiostatic control. The dashed curve was obtained with an O-ring electrode, while the solid curves represent results obtained with an electrode sealed with epoxy resin. The potential was scanned at a rate of $50 \mathrm{mV} / \mathrm{s}$.

electrode is low and comparable to the dark current in Fig. 7. When the electrode edges are exposed to the electrolyte, a significantly larger current is found, which shows a maximum similar to that of Fig. 1 and Fig. 2. At the same time, quite strong emission is observed in the visible region of the spectrum. In contrast to the luminescence observed with $p$-type porous silicon, the light is not emitted uniformly, but only near the electrode edges; the centre part of the electrode surface is inactive for the emission process.

A difference was also observed between the two types of electrode in galvanostatic operation. With the O-ring electrode more than $15 \mathrm{~V}$ was required to establish current densities as low as $1 \mathrm{~mA}$ $\mathrm{cm}^{-2}$, which indicates that the absence of holes in the $n$-type material hinders the anodic oxidation. Electrodes sealed with epoxy could be oxidized galvanostatically; results similar to those presented in Fig. 4 were obtained. However, the potential remained approximately constant for a longer time at values higher than the plateau values shown in Fig. 4. We also studied the luminescence from this electrode during the period of constant potential; again emission was only observed near the edges of the electrode.

\section{Discussion}

\subsection{Anodic oxidation and luminescence}

Oxidation of $p$-type porous silicon occurs in the dark as is clear from the considerable anodic current shown in Fig. 2a. On the other hand the dark current for n-type porous silicon is low. Clearly holes are required for the anodic oxidation of the porous semiconductor. This conclusion is supported by the experiments in which infrared light was used to generate charge carriers in the substrate. The electric field at the surface of the substrate separates the electrons and holes (see Fig. 11) and the holes can be injected into the porous structure.

There are two possible oxidation processes. The hole may be captured by an $\mathrm{Si}-\mathrm{H}$ surface bond,

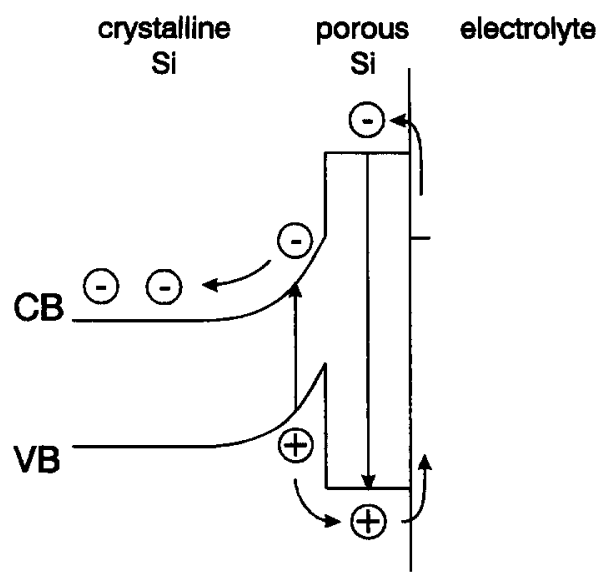

Fig. 11. Energy level diagram of an $n$-type porous silicon electrode in contact with an indifferent electrolyte. The origin of infrared induced oxidation and visible light emission is schematically represented. 


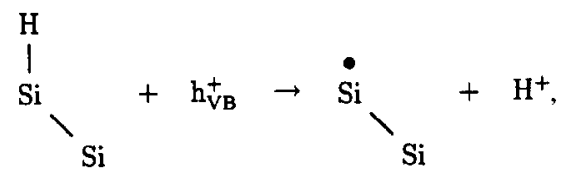

giving a radical intermediate (a dangling bond). The capture of a second hole leads to the formation of an $\mathrm{Si}-\mathrm{OH}$ species:

$$
\dot{\mathrm{Si}} \backslash_{\mathrm{Si}}+\mathbf{h}_{\mathrm{VB}}^{+}+\mathrm{H}_{2} \mathrm{O} \rightarrow \stackrel{\mathrm{Si}}{\mathrm{OH}_{\mathrm{Si}}}+\mathrm{H}^{+} .
$$

Another possibility is that the hole is trapped in an $\mathrm{Si}-\mathrm{Si}$ bond at the surface,

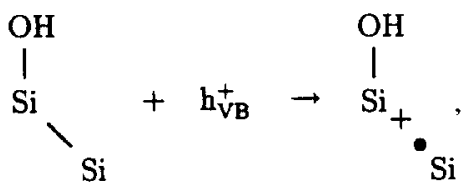

again forming a radical intermediate. The ruptured surface bond resulting from this reaction can react with water to form $\mathrm{Si}-\mathrm{OH}$ :<smiles>[13CH3][13CH2][Si]O</smiles>

The intermediate can undergo further oxidation to form silicon oxide by capturing a second hole from the valence band:

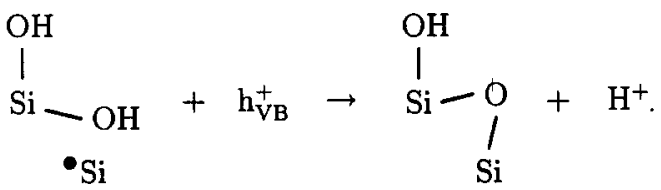

Reactions (4-6) can also occur with an $\mathrm{Si}-\mathrm{H}$ surface instead of an $\mathrm{Si}-\mathrm{OH}$ surface. It is likely that reactions (2-3) and reactions (4-6) are coupled [21]. If $\mathrm{Si}-\mathrm{H}$ is oxidized to $\mathrm{Si}-\mathrm{OH}$ the back bond is destabilized and reaction (4) should occur rapidly. If on the other hand the back bond is attacked first then the inclusion of oxygen destabilizes the $\mathrm{Si}-\mathrm{H}$ surface bond. Uosaki et al. [13] have shown that for porous silicon the decrease in the $\mathrm{Si}-\mathrm{H}$ coverage of the surface is accompanied by an increase in the $\mathrm{Si}-\mathrm{O}-\mathrm{Si}$ coverage.

The reactions mentioned so far account for the results of the potentiodynamic and galvanostatic experiments. However, as only hole capture is involved such a reaction scheme cannot explain the emission in $p$-type electrodes which clearly requires electron injection into the conduction band. The radical intermediates formed in reaction (2) or reaction (5) very likely have energy levels in the bandgap. Electrons in such levels may be thermally excited to the conduction band,

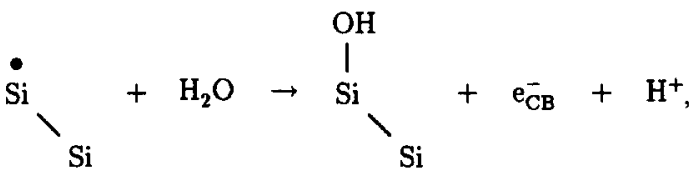

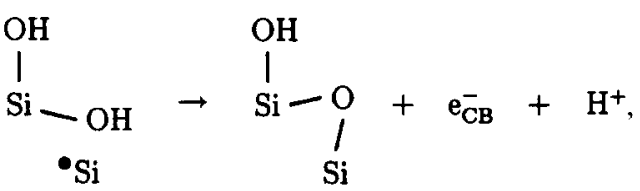

so that reactions (3) and (6) now proceed via the conduction band. Whether both electron injection steps take place, or only one, is not clear from our experiments. Moreover, the two intermediates are actually quite similar; in both cases a dangling bond is present on a silicon surface atom. A similar electron injection mechanism was proposed for the photoanodic dissolution of $n$-type silicon in solutions containing HF $[22,23]$. A hole is first captured at the surface. Subsequent electron injection from dissolution intermediates yield photocurrent quantum efficiencies of up to four; for each photon absorbed at least one hole and up to three electrons contribute to the current in the external circuit. Such a mechanism of competing electron and hole steps resembles that proposed for the anodic dissolution of $p$-type InP [17]; a hole is trapped in an In-P surface bond forming an intermediate state. This radical intermediate has an energy level within the bandgap near the conduction band edge, and electron injection can occur, which accounts for the emission detected during the anodic dissolution.

With this reaction scheme the visible luminescence of infrared illuminated $n$-type porous silicon can also be explained. In Fig. 11 the generation of emission is schematically shown. The photogenerated holes migrate towards the porous silicon/substrate interface and are transferred to the luminescent regions within the porous struc- 
ture, where oxidation leads to formation of intermediates (reactions (2) and (4)). Electrons are thermally excited to the conduction band via reaction (7) or (8), from which they undergo radiative recombination with photogenerated holes injected into the same luminescent regions.

\subsection{Stabilization}

From the results shown in Figs. 3 and 5 it is clear that the presence of ferrocyanide in solution suppresses the oxidation of porous silicon significantly. It is known that $\mathrm{Fe}(\mathrm{CN})_{6}^{4-}$ is able to stabilize crystalline silicon electrodes [18]. From a comparison of the standard potential of the $\mathrm{Fe}(\mathrm{CN})_{6}^{3-14-}$ redox couple in aqueous solution and the position of the band edges of crystalline silicon $(\mathrm{pH}=7)[24]$, it is clear that $\mathrm{Fe}(\mathrm{CN})_{6}^{4-}$ may be oxidized by capturing a hole directly from the valence band:

$$
\mathrm{Fe}(\mathrm{CN})_{6}^{4-}+\mathrm{h}_{\mathrm{VB}}^{+} \rightarrow \mathrm{Fe}(\mathrm{CN})_{6}^{3-}
$$

In a p-type semiconductor under anodic bias there is a large equilibrium surface concentration of holes. If reaction (9) is the only path for oxidation of $\mathrm{Fe}(\mathrm{CN})_{6}^{4-}$, then the rate of reaction (4) should not be influenced by $\mathrm{Fe}(\mathrm{CN})_{6}^{4-}$. To achieve stabilization, reaction (6) (or (8)) has to be suppressed. This is possible if the reducing agent injects an electron directly into the electron-deficient bond formed in reaction (4),

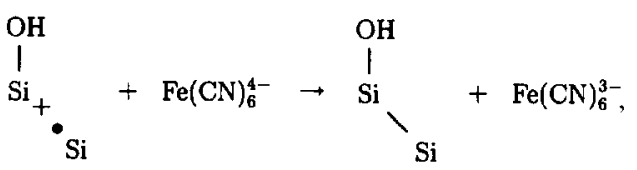

thereby leading to bond repair. This is again similar to the bond repair mechanism described by Schoenmakers et al. for the case of InP [17] and by Gomes et al. for GaAs and GaP [25-27]. Clearly, if bond repair via a reaction of the type shown in Eq. (10) is fast, then the surface density of bandgap states, from which an electron can be thermally excited to the conduction band, will be greatly reduced. Consequently, luminescence is quenched.

\subsection{Electrode edge effects}

The results of $n$-type electrodes with edges exposed to the solution are similar to those of $p$ type electrodes (Fig. 10); illumination is not needed for anodic oxidation. Similar results with $n$-type porous silicon electrodes have been reported by others $[9,10]$. The importance of crystallographic defects for the anodic oxidation of $n$-type silicon is nicely demonstrated in work by Heyboer et al. [28]. In experiments under chemomechanical polishing conditions they showed a correlation between defects caused by the polishing pad and the magnitude of the anodic current. In our experiments, defects in damaged regions at the electrode edges give rise to energy levels within the bandgap. Such defects can provide for a high generation rate of minority carriers, responsible for the oxidation of the silicon electrode. Holes are transferred to the porous layer where electron-deficient surface bonds are again formed (reaction (4)). Electrons injected from these intermediate states undergo radiative recombination with holes. The fact that the emission is mainly observed at the electrode edges indicates that the damaged areas are indeed the source of the minority carriers. When an O-ring is used to screen the edges from the solution, a low dark current without light emission is found.

\section{Conclusions}

We have studied the anodic oxidation and the corresponding luminescence of $p$-type and $n$-type porous silicon in indifferent electrolyte solutions. From both potentiodynamic and galvanostatic experiments it is found that the oxidation requires valence band holes at the surface. With $n$-type electrodes illuminated with infrared light and with $p$-type electrodes in the dark, strong visible emission is observed, indicating that electrons are injected during the formation of an oxide layer. Based on the results, a mechanism is proposed similar to that suggested for III-V semiconductors. A hole is trapped in an $\mathrm{Si}-\mathrm{H}$ or an $\mathrm{Si}-\mathrm{Si}$ surface bond, which leads to the formation of a radical intermediate. Due to the position of the energy levels associated with these radicals, electrons may 
be thermally excited to the conduction band where they undergo radiative recombination. The stabilization of the electrodes against passivation by the presence of ferrocyanide has also been studied. The oxidation is significantly delayed while the emission is quenched completely upon addition of $\mathrm{Fe}(\mathrm{CN})_{6}^{4-}$ to the solution. A bond repair mechanism involving electron injection into electrondeficient bonds by the reducing agent in solution is assumed to be responsible for the stabilization effects. From this work it is clear that the anodic luminescence from porous silicon is not due to a simple electroluminescence mechanism. The minority carriers are generated by an electrochemical reaction for which holes are first required.

\section{Acknowledgements}

The work described here was supported by the Netherlands Foundation for Chemical Research (SON) with financial aid from the Netherlands Organization for Scientific Research (NWO).

\section{References}

[1] P.M.M.C. Bressers, J.W.J. Knapen, E.A. Meulenkamp and J.J. Kelly, Appl. Phys. Lett. 61 (1992) 108.

[2] L.T. Canham, W.Y. Leong, M.I.J. Beale, T.I. Cox and L. Taylor, Appl. Phys. Lett. 61 (1992) 2563.

[3] E.S. Kooij, R.W. Despo and J.J. Kelly, Appl. Phys. Lett. $66(1995) 2552$.

[4] E.S. Kooij, R.W. Despo, F.P.J. Mulders and J.J. Kelly, J. Electroanal. Chem. 404 (1996) 139.

[5] A. Halimaoui, C. Oules, G. Bomchil, A. Bsiesy, F. Gaspard, R. Herino, M. Ligeon and F. Muller, Appl. Phys. Lett. 59 (1991) 304.

[6] A. Bsiesy, F. Gaspard, R. Herino, M. Ligeon, F. Muller and J.C. Oberlin, J. Electrochem. Soc. 138 (1991) 3450.

[7] M. Ligeon, F. Muller, R. Herino, F. Gaspard, J.C. Vial,
R. Romestain, S. Billat and A. Bsiesy, J. Appl. Phys. 74 (1993) 1265.

[8] F. Muller, R. Herino, M. Ligeon, F. Gaspard, R. Romestain, J.C. Vial and A. Bsiesy, J. Lumin. 57 (1993) 283.

[9] P.C. Searson, S.M. Prokes and O.J. Glembocki, J. Electrochem. Soc. 140 (1993) 3327.

[10] E.A. Meulenkamp, P.M.M.C. Bressers and J.J. Kelly, Appl. Surf. Sci. 64 (1993) 283.

[11] J.-N. Chazalviel and F. Ozanam, in: Microcrystalline Semiconductors: Materials Science and Devices, Eds. P.M. Fauchet, C.C. Tsai, L.T. Canham, I. Shimizu and Y. Aoyagi (Mater. Res. Soc. Proc. 283, Boston, USA, 1992) pp. 359-364.

[12] S. Billat, J. Electrochem. Soc. 143 (1996) 1055.

[13] K. Uosaki, T. Kondo, H. Noguchi, K. Murakoshi and Y.Y. Kim, J. Phys. Chem. 100 (1996) 4564.

[14] M.A. Hory, R. Herino, M. Ligeon, F. Muller, F. Gaspard, I. Mihalcescu and J.C. Vial, Thin Solid Films 255 (1995) 200.

[15] E. Landemark, C.J. Karlsson and R.I.G. Uhrberg, Phys. Rev. B 44 (1991) 1950.

[16] M. Buongiorno Nardelli, F. Finocchi, M. Palummo, R. Di Felice, C.M. Bertoni, F. Bernardini and S. Ossicini, Surf. Sci. 269/270 (1992) 879.

[17] G.H. Schoenmakers, R. Waagenaar and J.J. Kelly, J. Electrochem. Soc. 142 (1995) L60.

[18] M.J. Madou, K.W. Frese and S.R. Morrison, J. Phys. Chem. 84 (1980) 3423.

[19] W.J. Albery and M.L. Hitchman, Ring-disc Electrodes (Clarendon, Oxford, 1971).

[20] R.L. Smith and S.D. Collins, J. Appl. Phys. 71 (1992) R1.

[21] E.P. Boonekamp, J.J. Kelly, J. van der Ven and A.H.M. Sondag, J. Electroanal. Chem. 344 (1993) 187.

[22] H.J. Lewerenz, J. Stumper and L.M. Peter, Phys. Rev. Lett. 61 (1988) 1989.

[23] L.M. Peter, A.M. Borazio, H.J. Lewerenz and J. Stumper, J. Electroanal. Chem. 290 (1990) 229.

[24] M.J. Madou, B.H. Loo, K.W. Frese and S.R. Morrison, Surf. Sci. 108 (1981) 135.

[25] Lu Shou Yun, D. Vanmaekelbergh, W.P. Gomes and F. Cardon, Ber. Bunsenges. Phys. Chem. 91 (1987) 390.

[26] D. Vanmaekelbergh, Lu Shou Yun, W.P. Gomes and F. Cardon, J. Electroanal. Chem. 221 (1987) 187.

[27] W.P. Gomes, S. Lingier and D. Vanmaekelbergh, J. Electroanal. Chem. 269 (1989) 237.

[28] W.L.C.M. Heyboer, G.A.C.M. Spiering and J.E.A.M. van den Meerakker, J. Electrochem. Soc. 138 (1991) 774. 